\title{
Improved Dynamic Response Assessment for Intra-Articular Injected Iron Oxide Nanoparticles
}

\author{
L. A. Crowe ${ }^{1 \star}$ F. Tobalem, ${ }^{1}$ A. Gramoun, ${ }^{1}$ B. M. A. Delattre,${ }^{1}$ K. Grosdemange, ${ }^{1}$ \\ J. Salaklang, ${ }^{2}$ A. Redjem, ${ }^{2}$ A. Petri-Fink, ${ }^{2}$ H. Hofmann, ${ }^{3}$ and J.-P. Vallée ${ }^{1}$
}

\begin{abstract}
The emerging importance of nanoparticle technology, including iron oxide nanoparticles for monitoring development, progression, and treatment of inflammatory diseases such as arthritis, drives development of imaging techniques. Studies require an imaging protocol that is sensitive and quantifiable for the detection of iron oxide over a wide range of concentrations. Conventional signal loss measurements of iron oxide nanoparticle containing tissues saturate at medium concentrations and show a nonlinear/nonproportional intensity to concentration profile due to the competing effects of $T_{1}$ and $T_{2}$ relaxation. A concentration calibration phantom and an in vivo study of intra-articular injection in a rat knee of known concentrations of iron oxide were assessed using the difference-ultrashort echo time sequence giving a positive, quantifiable, unambiguous iron signal and monotonic, increasing concentration response over a wide concentration range in the phantom with limited susceptibility artifacts and high contrast in vivo to all other tissues. This improved dynamic response to concentration opens possibilities for quantification due to its linear nature at physiologically relevant concentrations. Magn Reson Med 68:1544-1552, 2012. (c) 2012 Wiley Periodicals, Inc.
\end{abstract}

Key words: SPION, iron oxide nanoparticles, ultrashort echo, concentration, intra-articular

Early diagnostic tools and biomarkers for the detection and determination of disease onset and for evaluating the rapidity of disease progression as well as the ability to gain rapid insight into the efficacy of therapeutic interventions are essential if future drug development and nanotechnology are to progress. Significant research is reported in "theranostic" applications, in which molecular imaging is exploited for both detection of disease

\footnotetext{
${ }^{1}$ Division of Radiology, Geneva University Hospitals, University of Geneva, Faculty of Medicine, Foundation for Medical Researchers, Geneva, Switzerland.

${ }^{2}$ Adolphe Merkle Institute, University of Fribourg, Fribourg, Switzerland.

${ }^{3}$ Powder Technology Laboratory, Ecole Polytechnique Fédérale de Lausanne, Lausanne, Switzerland.

Grant sponsor: European Commission (7th Framework Programme for Research "Nanosciences, Nanotechnologies, Materials and New Production Technologies”); Grant number: NMP4-LA-2009-228929; Development of Novel Nanotechnology Based Diagnostic Systems for Rheumatoid Arthritis and Osteoarthritis (NanoDiaRA); Grant sponsors: Centre for Biomedical Imaging (CIBM) of the Geneva, Lausanne, Leenaards, Louis-Jeantet and Insuleman Foundations

${ }^{*}$ Correspondence to: L. A. Crowe, PhD, Division of Radiology, Geneva University Hospitals, Rue Gabrielle-Perret-Gentil 4, 1211 Geneva, Switzerland. E-mail: lindsey.crowe@hcuge.ch
}

and delivery of treatment $(1,2)$. Existing diagnostic methods, namely X-ray-based techniques and magnetic resonance imaging (MRI), can detect disease outcomes such as in rheumatoid arthritis by visualizing structural damage. What is needed is the ability to detect and measure the disease process rather than morphological modification, so we can obtain earlier diagnosis or improved treatment monitoring. By using MRI-based molecular imaging of injected iron oxide nanoparticles (IONs) it should be possible to detect experimental and human arthritis onset early and enable progression monitoring of the disease and treatment responses much earlier and more effectively, thereby minimizing irreversible joint damage. There is an important need for the development of rheumatoid arthritis-targeted IONs for either intravenous or intra-articular (IA) injection.

Targeted molecular treatment, and its evolution with time, needs highly sensitive and quantifiable detection of the carrier iron oxide particles. The presence of superparamagnetic IONs (SPIONs) with the core size of 8-10 $\mathrm{nm}$ produces strong localized regions of signal loss on conventional MR images by destroying the homogeneity of the surrounding magnetic field. Preliminary reports suggest the potential of MR imaging for noninvasive serial monitoring, for example, in animal models of arthritis (3), with cells labeled ex vivo including immune rejection (4) and similar applications (5-7), and in vivo uptake in inflammation and macrophage tracking (8). Despite the clear visibility of signal loss on the images, this cannot easily be quantified $(9,10)$. Problems include similar areas of hypointense signal that can be created by other structures in the same region, inhomogeneous background signal, and complete saturation of the signalto-noise levels after an initial peak in intensity at very low concentrations. MR sequences for positive contrast rather than hypointense signal for iron-loaded tissue have been the subject of intense research, as positive contrast methods improve the detection of iron oxide-labeled tissue and may help to solve quantification difficulties. The advantages and limitations of these positive contrast methods for iron oxide have been reported in the literature. These include inversion recovery with onresonant water suppression (11), susceptibility gradient mapping (12), white-marker GRe Acquisition for Superparamagnetic Particles (GRASP) (13), and phase map cross correlation (14). Limitations of inversion recovery with on-resonant water suppression include loss of anatomical information, necessity for projection reconstruction, off-resonance lipid effects, and sensitivity to largescale $B_{0}$ inhomogeneity. White-marker techniques and retrospective phase map cross correlation depend on the orientation of the susceptibility effect with respect to $B_{0}$. 
These positive contrast techniques have been shown ex vivo, on $2 \mathrm{D}$ images, and with distorted signal patterns in relation to true anatomy (15-17). A standard positive contrast method has not yet emerged. Recently, ultrashort echo time (UTE) imaging $(18,19)$ has been proposed as a technique for enhancement of localized regions of iron uptake (20), and a three-dimensional (3D) radial UTE sequence has been reported for cellular, brain, and skeletal muscle imaging (21-24). Three-dimensional UTE imaging (23) uses nonselective radiofrequency and radial sampling from the centre of $k$-space, with short read out and TE (24). A UTE image includes signal from all tissues, including those with very short $T^{*}{ }_{2}$ because of the presence of iron. A second echo acquired after short $T^{*}{ }_{2}$ decay is subtracted giving positive contrast from the ironcontaining tissue and uniform nulled background. The difference-ultrashort echo time (dUTE) methods not only give positive iron signal but also allow easy localization of the signal due to maintaining background anatomy in the individual echoes with 3D isotropic resolution. dUTE does not require retrospective correction and is not subject to large susceptibility effects or fat/water shifts (18). Therefore, UTE appears to be the sequence of choice for imaging IONs. In addition to the desired TE properties, radial imaging has a diffuse distribution of artifacts with no wraparound, making it robust to flow and motion, and ideal for small field-of-view (FOV) imaging.

A major problem in iron oxide imaging is quantification of the resulting signal. Currently, little is known about the performance of dUTE for the quantification of iron oxide concentration, which remains extremely challenging regarding the competing effects of relaxation times. UTE provides signal from the shortest $T_{2}$ species giving positive signal from iron, and dUTE shows unambiguous iron concentration response (20). $T_{1}$ effects increase signal intensity at low concentrations, followed by signal loss at mid to high concentrations (or in the case of clustering of particles) when $T^{*}{ }_{2}$ dephasing signal loss becomes dominant $(25,26)$. The competing effects of shortened $T_{1}$ and $T_{2}$ will increase and decrease signal intensity, respectively. These effects must be separated by a sequence sensitive to either $T_{1}$ or $T_{2}$. By taking an UTE ( $T_{1}$ or proton density-like image), and one that slightly longer, but still classically very short $\left(T_{2}\right)$, the effects of the relaxation times will be isolated. The signal remaining from even the shortest $T_{2}$ species can then be quantified using the dUTE approach.

With dUTE we, therefore, can report two related advantages: the $T_{2}$ effect in the UTE echo is isolated from other relaxation effects by the use of a second echo, and the information about species, such as those containing iron oxide, with very short $T_{2}$ is quantifiable because of the lack of signal loss in the UTE echo. As a precursor to studies of ION uptake in arthritis, the dose response of new positive iron oxide contrast MR imaging sequences and conventional MR imaging sequences was investigated in vitro using a calibration phantom and in vivo by IA injection in normal rat knees. After phantom calibrations, the IA injected model was chosen as a method to obtain in vivo images with a known concentration of SPIONs as it is difficult to quantify iron oxide in tissues after intravenous injection. This study serves as a rationale for "theranostic" studies in arthritis models with local delivery of drugs coupled to nanoparticles and for particles targeted to, for example, cartilage degradation via antibodies $(1,3,8,27)$. Both noninvasive quantification of the amount of a targeted particle reaching an anatomical location and the monitoring of kinetics for local injection are key elements in optimizing emerging nanoparticle studies.

\section{METHODS}

\section{Particle Production}

The SPIONs were manufactured by an aqueous coprecipitation method, as described previously (28). The colloidal particles were further coated with a mixture of poly (vinyl alcohol) (PVA) and vinyl alcohol/vinyl amine copolymer. Therefore, the particle suspension was mixed with a solution of the polymer mixture at a weight ratio particle/polymer of 1:9. The product will be referred to as A-PVA-SPION in this work. The iron content of the suspensions was determined by redox titration.

All chemicals were of analytical reagent grade and were used without further purification. PVA (Mowiol ${ }^{\circledR}$ 3-85) with an average molecular weight of 14,000 g/mol and a hydrolysis degree of $85 \%$ was supplied by courtesy of CLARIANT (Muttenz, Switzerland). Vinyl alcohol/vinyl amine copolymer M12, with an average molecular weight of 80,000-140,000, was supplied by courtesy of ERKOL (Tarragona, Spain).

\section{Dye Coupling}

Surface derivatization of polymer-coated particles was performed in the previously described magnetic bed reactor (29). Therefore, A-PVA-SPIONs were loaded and immobilized in the reactor. Dye coupling was achieved by recirculating $100 \mu \mathrm{g}$ FluoProbes (FluoProbes®682, Interchim, Montlucon, France) in $0.1 \mathrm{M}$ carbonate buffer ( $\mathrm{pH}$ 8.3) over the immobilized nanoparticles for $2 \mathrm{~h}$ with a flow rate of $1.0 \mathrm{~mL} / \mathrm{min}$. Finally, the functionalized particles were eluted from the magnetic reactor, redispersed in PBS, and characterized (Table 1). Because of technical difficulties, the fluorophore was not used in this particular study but did not significantly change the behavior of the particles in this application.

\section{Transmission Electron Microscopy}

Transmission electron microscopy was performed using a Phillips (Koninklijke, Netherlands) CM-20 microscope operating at $200 \mathrm{kV}$. For sample preparation, dilute drops of suspensions were allowed to dry slowly on carbon-coated copper grids.

\section{Particle Size}

Light scattering measurements were carried out at $90^{\circ}$ on a photon correlation spectrometer from Brookhaven (Holzville, NY) equipped with a BI-9000AT digital autocorrelator. The CONTIN method was used for data processing. The concentration of IONs was set to $100 \mu \mathrm{g}$ iron $/ \mathrm{mL}$ for all measurements. The theoretical refractive index of 2.42 of magnetite was used to calculate the number-weighted 
Table 1

Particle Properties Before and After Dye Coupling

\begin{tabular}{|c|c|c|c|c|c|}
\hline Particle & $\begin{array}{l}\text { Particle } \\
\text { size }(n m)\end{array}$ & $\begin{array}{l}\text { Zeta potential } \\
\qquad(\mathrm{mV})\end{array}$ & $\begin{array}{c}\text { No. of dye } \\
\text { molecules/particle }\end{array}$ & $\begin{array}{l}\text { Dispersing } \\
\text { medium }\end{array}$ & $\begin{array}{l}\text { Iron concentration } \\
\text { (mg Fe/mL) }\end{array}$ \\
\hline A-PVA-SPIONs & $38.4 \pm 3.4$ & $24.4 \pm 5.3$ & - & Water & 5 \\
\hline $\begin{array}{l}\text { FluoProbes }{ }^{\circledR} 682 \text { conjugated } \\
\text { A-PVA-SPIONs }\end{array}$ & $48.9 \pm 4.6$ & $-4.6 \pm 0.6$ & 7.2 & PBS & 2 \\
\hline
\end{tabular}

distribution from the raw intensity-weighted data. Viscosity, refractive index, and dielectric constant of pure water were used to characterize the solvent.

\section{Phantom Calibration}

A phantom was constructed with concentrations ranging from 12.5 to $200 \mu \mathrm{g} / \mathrm{mL}$ plus one water. Concentrations are given throughout in microgram of iron per milliliter of solution (in physiological $\mathrm{NaCl}$ ). Scanning was carried out on a Siemens Magnetom Trio, a Tim system, 3 T clinical scanner (Siemens AG, Erlangen, Germany) with the manufacturer's 4-cm loop coil. The protocol parameters included 3D $T_{1}$ gradient echo (named in the article as the Siemens acronym VIBE), with parameters repetition time (TR)/TE 14.3/5.9 ms, flip angle $12^{\circ}$, fat suppression, isotropic resolution $0.31 \mathrm{~mm}$, and FOV. dUTE consisted of the acquisition and subtraction of two TEs (ultrashort UTE and short TE2) leading to positive contrast from short $T_{2}$ species and reduced signal elsewhere. Parameters here are 3D isotropic resolution matrix of 448 and $80 \mathrm{~mm}$ FOV, giving a resolution of $0.18 \mathrm{~mm}$ in all three acquired directions allowing freedom of reconstructed image planes, 50,000 radial projections, UTE/TE2 $0.07 \mathrm{~ms} / 2.46$ $\mathrm{ms}$ (for in-phase fat/water image), TR $9.6 \mathrm{~ms}$, and flip angle $10^{\circ}$. All phantom tubes were scanned in the same acquisition and, later, bandwidth and receiver gain were kept constant for the IA in vivo images.

This was followed by a short TI inversion recovery (TI $=$ inversion time) (STIR) 2D multislice spin echo (SE) sequence with TR/TE $3700 / 20 \mathrm{~ms}$, flip angle $150^{\circ}$, resolution $0.156 \mathrm{~mm}$, FOV $60 \mathrm{~m}$, and slice thickness $1 \mathrm{~mm}$. The STIR sequence was used for comparison, as it is the reference sequence for in vivo detection of edema. Also included here was a third echo to use with UTE for another dUTE image from subtraction of TE3 $=4.92 \mathrm{~ms}$ from the UTE echo to investigate the effect of TE on the dUTE contrast.

The dUTE experiment was repeated at $1.5 \mathrm{~T}$ on a Siemens Espree for comparison, with parameters as close as allowed by the different hardware. These were 3D radial with 50,000 radial projections, UTE/TE2/TE3 0.07 ms/ $2.46 \mathrm{~ms} / 4.92 \mathrm{~ms}$, TR $9.6 \mathrm{~ms}$, and flip angle $10^{\circ}$.

\section{Animal Handling and Imaging}

Female Lewis rats (Janvier, Le Genest-St-Isle, France), weighing 150-175 g (2 months old), were used throughout this study. Fifteen rats were scanned immediately after IA injection in the knee of IONs (A-PVA-SPION) at five different concentrations. Scanning was carried out on a Siemens Magnetom Trio, a Tim system, 3 T clinical scanner using the manufacturer's 4-cm loop coil. The protocol parameters included $3 \mathrm{D} T_{1}$ gradient echo (named in the article as the Siemens acronym VIBE), with parameters TR/TE $14.3 / 5.9 \mathrm{~ms}$, flip angle $12^{\circ}$, fat suppression, isotropic resolution $0.31 \mathrm{~mm}$, and FOV 100 $\mathrm{mm}$ to compare with previously published work (3) and used in further applications of diseased knees for bone erosion. dUTE consisted of the acquisition and subtraction of two TEs (ultrashort UTE and short TE2) leading to positive contrast from short $T_{2}$ species and reduced signal elsewhere. Parameters here are $3 \mathrm{D}$ isotropic resolution matrix of 448 and $80 \mathrm{~mm}$ FOV, giving a resolution of $0.18 \mathrm{~mm}$ in all three acquired directions allowing freedom of reconstructed image planes, 50,000 radial projections, UTE/TE2 $0.07 \mathrm{~ms} / 2.46 \mathrm{~ms}$ (for in-phase fat/water image), TR $9.6 \mathrm{~ms}$, and flip angle $10^{\circ}$.

Concentrations in microgram of iron per milliliter of solution (in physiological $\mathrm{NaCl}$ ) ranging from 12.5 to 200 $\mu \mathrm{g} / \mathrm{mL}$ were injected for the in vivo experiments using $50 \mu \mathrm{L}$ per knee. Concentrations were chosen to cover as wide a range as possible after the phantom experiment and to be realistically low so as to represent iron than may reach the joint after systemic administration of iron particles taking into account average blood volume.

MR imaging was carried out immediately after injection. The suturing of the joint, installation of the animal in the MRI coil, and pilot scan took $4 \mathrm{~min}$. The images were then acquired within the next $40 \mathrm{~min}$.

Animals were anesthetized with inhaled isoflurane during imaging and monitored with a respiratory pad (SA Instruments, Stony Brook, NY).

\section{Image Analysis}

For both phantom and in vivo images, regions were drawn blinded with respect to concentration to quantify the intensity of the signal in the regions containing iron particles. For the in vivo case, homogeneous regions within the region of iron distribution in the capsule in the infrapatellar pouch were chosen. Standard deviation error bars (95\% confidence) are plotted for the mean measurements over several animals.

Numerical analysis was carried out using analysis of variance (ANOVA) with post hoc Bonferroni (PASWStatistics SPSS Inc. (Chicago, IL) 18.0) and a $P<0.05$ was set as significant.

Ethical committee approval was obtained for the complete protocol, and animals were kept in the institutions animal facility with free access to food and water.

\section{RESULTS}

\section{Phantom Calibration Curves}

Phantom results allow us to assess the utility of the different sequences for quantifying an iron concentration effect (Fig. 1a). At very low iron concentrations, a $T_{1}$ 

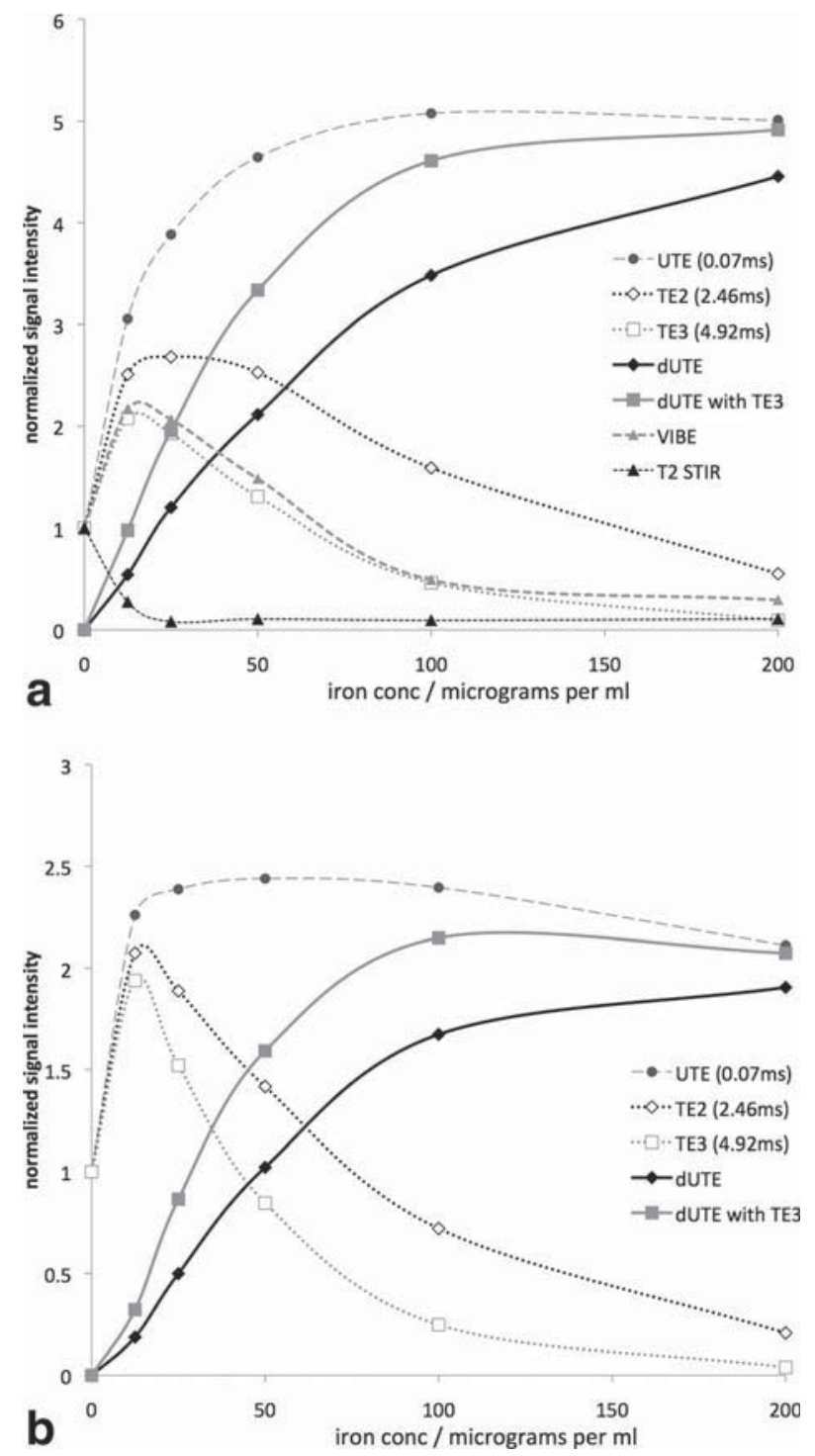

FIG. 1. Phantom results. Comparison of normalized signal intensity versus concentration of iron oxide in sample tubes, normalized to the signal at concentration 0 (water). Proposed dUTE method and conventional signal loss sequences comparing sensitivity and ambiguity at different concentrations. Normalized signal intensity as a function of second echo time for the dUTE method showing monotonic concentration dynamic of dUTE. a: 3 T. b: $1.5 \mathrm{~T}$

effect dominates in all the gradient-echo MR sequences including even the UTE sequence and hyperintense signal appears. At high iron concentrations, $T^{*}{ }_{2}$ effects induce a signal loss on all the images with the exception of the UTE images. The resulting peak at low concentrations in the 3D GRE and second echoes (TE2 and TE3) means ambiguity in calibration. These signal loss images also saturate to zero signal-to-noise ratio (SNR) at concentrations above $100 \mu \mathrm{g} / \mathrm{mL}$, whereas the dUTE image continues to distinguish concentrations twice this value. Only with the dUTE image with a short second TE does the monotonic increasing relation with concentration become apparent. A longer second echo (TE3) in the dUTE protocol gives a steeper initial slope, hence higher sensitivity, but saturates at lower concentrations leading to less linearity. Further problems are encountered in vivo with this longer TE3 and are discussed below. The difference image with the shorter second echo has a smaller slope at low concentrations, but much better linearity over a longer range. Up to $200 \mu \mathrm{g} / \mathrm{mL}$ of iron, the linear fit of dUTE $R^{2}$ is 0.90 for the TE2 and 0.67 for the TE3. At physiologically relevant concentrations up to $100 \mu \mathrm{g} / \mathrm{mL}$, the linear fit for dUTE is excellent with an $R^{2}$ of 0.99 . The advantage of the subtraction method is shown by these statistics to $100 \mu \mathrm{g} / \mathrm{mL}$, as for the UTE, second and third echoes of the $3 \mathrm{D}$ radial sequence, the $R^{2} / p$ of linear regression are $0.698 / 0.078,0.001 / 0.969$, and $0.420 / 0.237$, with only the subtraction dUTE showing a significant $P<0.002\left(R^{2}=0.994\right)$ with the shorter second echo, which is slightly better than using a longer second echo, TE3 (0.967/0.003) which only performs marginally better up to $50 \mu \mathrm{g} / \mathrm{mL}(0.995 / 0.05$ for dUTE with TE2 and 0.996/0.04 for dUTE with TE3) due to the steeper initial linear slope but earlier signal saturation. By Fisher test, the slopes for dUTE with both TE2 and TE3 are significant compared with the regression of the single echoes. Similar results are obtained at $1.5 \mathrm{~T}$. By comparison to the gradient-echo MR sequences, the spin echo-based STIR sequence was characterized by a signal drop in the synovial cavity at all the iron concentrations as well as an early signal saturation for concentrations more than $25 \mu \mathrm{g} / \mathrm{mL}$ of iron.

At $1.5 \mathrm{~T}$, the dUTE curve is very similar to the curve obtained at $3 \mathrm{~T}$, with a slightly lower sensitivity (slope) at low concentrations and is closer to linearity at the high concentration end of the range. At $1.5 \mathrm{~T}$, very similar behavior is seen as at $3 \mathrm{~T}$, as the subtraction of dUTE relies on the interplay of $T_{1}$ and $T_{2}$, both of which are affected by the change in field. The sensitivity to $T_{2}$ is to be considered, but the advantages at the higher field are more important than the slight changes in concentration response. For the dUTE sequence, there was not an observable difference in signal behavior at the two fields due to the subtraction of images and the interplay of $T_{1}$ and $T_{2}$ effects. There was, however, with scan parameters as close as possible with the differing hardware, an advantage of around 1.4 in SNR. The plots are shown in Fig. 1b. The $T_{2}$ STIR drops to the noise level even at the lowest concentration of $12.5 \mu \mathrm{g} / \mathrm{mL}$.

\section{In Vivo Images}

In vivo images (Fig. 2) show the iron oxide in the knee joint; however, at high concentrations, susceptibility artifacts (hypointense signal) become evident even in the UTE image in the regions of high iron concentration in the synovium (see UTE $200 \mu \mathrm{g} / \mathrm{mL}$ image in Fig. 2). However, these concentrations are unlikely to be reached with systemic injection. The VIBE sequence clearly shows its ambiguity with high signal intensity at 12.5 $\mu \mathrm{g} / \mathrm{mL}$ followed by signal loss. The dUTE difference image removes this competing effect of $T_{1}$ and $T_{2}$ (30), where a similar response to iron concentration is seen for the phantom and in vivo results.

In vivo concentration effects depend on the parameters of the different sequences. For quantification purposes, the uniform region of iron signal in the synovium is 

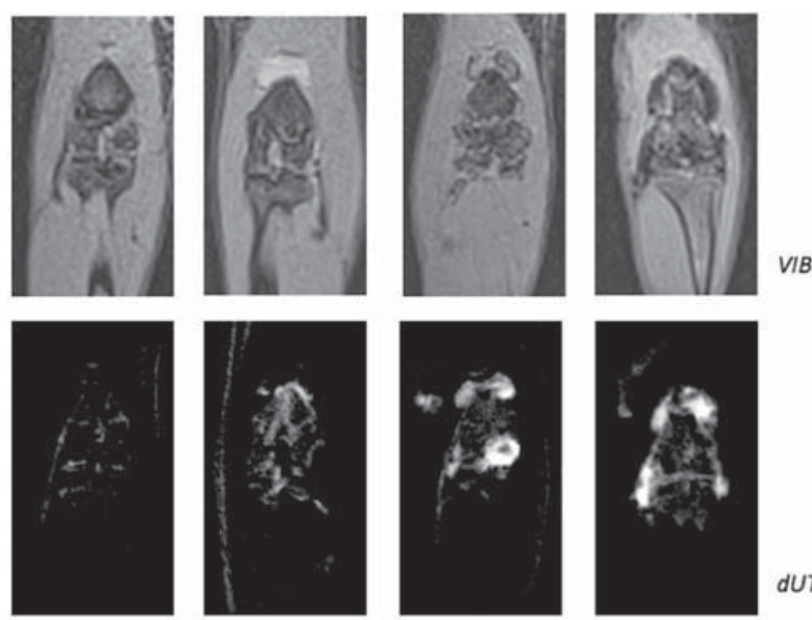

dUTE
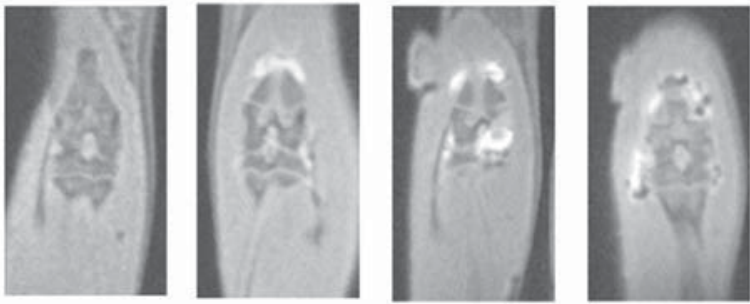

UTE

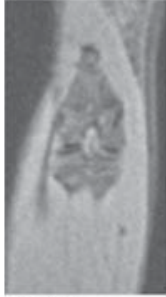

control

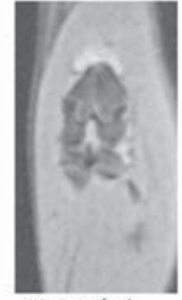

$12.5 \mu \mathrm{g} / \mathrm{ml}$

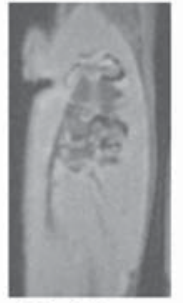

$50 \mu \mathrm{g} / \mathrm{ml}$

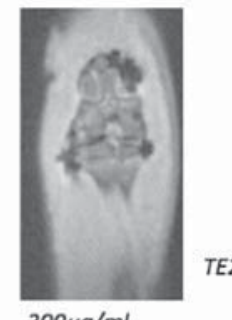

$200 \mu \mathrm{g} / \mathrm{ml}$
FIG. 2. In vivo results. Synovial iron on VIBE, dUTE, UTE, and TE2 images of a control and at $50 \mu \mathrm{L}$ volume intravenous injection of $12.5,50$, and $200 \mu \mathrm{g} / \mathrm{mL}$.

measured; however, the concentration correlation, the presence of susceptibility artifacts, and their effect of expanding the apparent size of the region of iron uptake in the VIBE sequence must be taken into account in assessing the utility of each sequence. There is poorer suppression of background in in-vivo imaging and more errors due to increased susceptibility artifact in the second echo if the TE is too long, reducing the feasibility for quantification. Table 2 gives contrast (signal difference) and signal intensity mean values for each concentration and sequence. Statistical analysis shows contrast values and their significance in separating different concentrations, where $P$ values are calculated for all the sequences separately. A second analysis uses the signal intensity values to assess whether any iron (grouping all the different concentrations together) can be distinguished from the surrounding tissue in each of the sequences described. The positive contrast overlaid on the VIBE signal loss sequence is shown in Fig. 3 with increasing concentration. The multiple locations at different levels of intensity for the different concentrations are clearly seen.

Figure 4 shows the comparison of the in vivo signal intensities measured in the homogeneous region of the infrapatellar synovium on the sequences at different concentrations. Here, the following observations for concentration effects and tissue contrast can be made if we note the separation and linearity of the iron signal from the other tissues in the dUTE plot.

For the separation of different iron concentrations, dUTE shows the expected monotonic correlation over the range studied. VIBE signal loss shows higher sensitivity at low concentrations, but saturates at a lower value, precluding distinction of higher concentrations. The VIBE iron signal also overlaps with other tissues reducing contrast to zero at certain mid range concentrations. The first echo UTE shows contrast of iron from the other tissues but does not have the clear proportionality with concentration. The in vivo intensities correlate with concentration for dUTE with a linear fit $\left(R^{2}=0.69\right.$ compared with 0.49 for an exponential fit), whereas VIBE fits better to the exponential $\left(R^{2}=0.79\right.$ for the exponential fit compared with 0.63 for a linear fit). The $R^{2}$ fits show that the dUTE fits well to a continuously increasing function in agreement with the phantom calibration results. VIBE always includes positive, negative, and zero contrast as we can see from the crossing lines on the signal intensity plots. dUTE contrast always same sign, and therefore there are no concentrations at which the iron signal is coincident with the surrounding muscle signal.

The further advantage of dUTE becomes apparent by comparing the contrast with the different tissues. The iron region signal is always separated from the muscle signal, and also, at all but the very lowest concentration, from the bone signal. This allows easy distinction of the size of the region of iron uptake from the surrounding area. This is not the case for any of the classical sequences compared or the simple single echo of the UTE acquisition. For VIBE, some low to intermediate concentrations show no contrast from muscle, similarly for the second and third echo of the dUTE method. This is shown in Fig. 5 comparing contrast-to-noise ratio between synovial iron and bone or muscle for the VIBE and dUTE images as a function of concentration indicating the relative signal contrast and dynamic between the different methods. Normalization of the values was done to the noise level for calculating contrast to noise ratio (CNR) as scaling to muscle tissue causes very different results in the two sequences due to the bright muscle signal in VIBE compared with the noise level muscle signal in dUTE.

Taking all the conditions together $(n=15)$, ANOVA (with post hoc Bonferroni) shows that only dUTE shows significant difference between signal for synovial iron, bone, and muscle in all cases $(P<0.013)$. Results from ANOVA show contrast and separation of surrounding tissue signal intensity and the different concentrations of iron. For illustrative purposes, with $P<0.05$ as statistically significant, there is an observed difference between all concentrations using dUTE, but not the lower concentrations using VIBE. Comparing regions of muscle or bone at different iron concentrations shows no significant difference. We observe that contrast between iron and bone or muscle is only different for dUTE, where both bone and muscle signals are suppressed. 
Table 2

Absolute Signal Intensity and Contrast Values (Defined as the Difference Between Iron and Tissue Signal Not Scaled to Noise) for Synovial Iron Regions and Other Tissues with Statistics for Distinction of Different Concentrations of Iron and the Advantages of dUTE for Distinction of Any Iron Concentration Signal from All Other Surrounding Tissues

\begin{tabular}{|c|c|c|c|c|c|c|c|c|c|}
\hline & \multirow[b]{2}{*}{ Conc $(\mu \mathrm{g} / \mathrm{mL})$} & \multicolumn{4}{|c|}{ Signal intensity ${ }^{a}$} & \multicolumn{4}{|c|}{$\begin{array}{l}\text { Contrast between synovial } \\
\text { iron and other tissues }\end{array}$} \\
\hline & & dUTE & VIBE & UTE & TE2 & dUTE* & VIBE $^{\star}$ & UTE $^{*}$ & TE2* \\
\hline \multirow[t]{5}{*}{ Synovial iron } & 12.5 & 34.47 & 381.19 & 173.19 & 158.74 & & & & \\
\hline & 25 & 83.51 & 301.84 & 196.86 & 111.43 & & & & \\
\hline & 50 & 101.13 & 80.54 & 176.74 & 77.07 & & & & \\
\hline & 125 & 115.53 & 32.90 & 138.39 & 14.02 & & & & \\
\hline & 200 & 141.97 & 26.92 & 155.22 & 11.40 & & & & \\
\hline \multirow[t]{5}{*}{ Bone } & 12.5 & 37.24 & 74.41 & 91.70 & 63.64 & -2.77 & 306.79 & 81.49 & 95.10 \\
\hline & 25 & 27.98 & 84.61 & 91.66 & 66.63 & 55.53 & 217.23 & 105.20 & 44.80 \\
\hline & 50 & 23.73 & 73.61 & 82.48 & 58.47 & 77.40 & 6.93 & 94.26 & 18.60 \\
\hline & 125 & 14.94 & 110.37 & 93.07 & 80.79 & 100.59 & -77.47 & 45.32 & -66.77 \\
\hline & 200 & 24.68 & 95.87 & 108.26 & 76.42 & 117.29 & -68.95 & 46.96 & -65.03 \\
\hline \multirow[t]{5}{*}{ Muscle } & 12.5 & 4.66 & 263.16 & 125.85 & 119.18 & 29.81 & 118.03 & 47.34 & 39.56 \\
\hline & 25 & 1.89 & 303.32 & 129.37 & 128.77 & 81.62 & -1.48 & 67.49 & -17.34 \\
\hline & 50 & -2.63 & 208.63 & 92.14 & 99.67 & 103.76 & -128.09 & 84.60 & -22.59 \\
\hline & 125 & -3.52 & 240.17 & 100.80 & 99.98 & 119.05 & -207.27 & 37.59 & -85.95 \\
\hline & 200 & -1.97 & 196.92 & 105.62 & 103.01 & 143.94 & -170.00 & 49.60 & -91.62 \\
\hline \multirow[t]{5}{*}{ Noise } & 12.5 & -6.03 & 15.62 & 4.52 & 5.94 & 40.50 & 365.57 & 168.67 & 152.79 \\
\hline & 25 & -0.72 & 15.99 & 4.68 & 5.39 & 84.23 & 285.85 & 192.18 & 106.04 \\
\hline & 50 & 0.35 & 13.00 & 4.84 & 4.79 & 100.78 & 67.54 & 171.90 & 72.28 \\
\hline & 125 & 0.10 & 15.88 & 4.57 & 4.53 & 115.44 & 17.02 & 133.83 & 9.49 \\
\hline & 200 & 0.93 & 14.42 & 5.18 & 5.05 & 141.04 & 12.50 & 150.04 & 6.35 \\
\hline
\end{tabular}

a Taking all the concentrations together to assess which sequence best separates any iron signal from other tissue, ANOVA (with post hoc Bonferroni) with $n=15$ shows the following:

- dUTE shows significant difference between signal for all synovial iron concentrations compared with surrounding bone and muscle (all $P<0.013$ ).

- For VIBE there is only signal difference between bone and muscle. For synovial iron signal difference to other tissues $P>0.1$, meaning iron is not separated from surrounding tissue in all cases.

- UTE has no significant difference between bone and muscle signal $(P>0.5)$.

- TE(2) shows no significant difference between synovial iron and bone, with a $P<0.04$ for the significant difference between synovial iron and muscle signal.

${ }^{*} P<0.01$ for contrast values comparing the different concentrations (for all values in bold). UTE synovial iron vs. noise (in italics) does not show a significant difference between iron concentrations. This represents the ability of dUTE and VIBE to distinguish different concentrations of iron.

\section{DISCUSSION AND CONCLUSIONS}

The main conclusions drawn from this study are as follows: dUTE gives a monotonic signal increase over a greater dynamic range of concentrations than for the other gradient-echo MR sequences including the UTE sequence (ultrashort echo on its own). dUTE shows a linear response of the MR signal as a function of iron oxide concentration up to a physiologically relevant concentration of $100 \mu \mathrm{g} / \mathrm{mL}$. This linear response is further validated in vivo using a rat model of IA injections of increasing iron oxide concentration.

Low iron oxide concentrations are detectable and quantifiable without ambiguity, and the signal intensity plateau (saturation) occurs at higher concentration than signal loss/VIBE sequences. As patient doses of iron oxide particles can be up to $8 \mathrm{mg} / \mathrm{kg}$ this would translate to around $2 \mathrm{mg}$ per rat. This would make a local $(1 \mathrm{~mL}$ volume) of 12.5-200 $\mu \mathrm{g}$ a reasonable value. Also with up to $14 \mathrm{~mL}$ of contrast agent injected in patients for arthroscopy, the volume of $50 \mu \mathrm{L}$ is of the same order of rela- tive magnitude. Both the volume and concentration are significantly lower than those used in previous animal studies, for example, $0.5 \mathrm{~mL}$ of $10 \mathrm{mg} / \mathrm{mL}$ solution in mice (31). There is a trade-off between the desired low concentration sensitivity and the improved dynamic response.

Three different TEs were tested with the radial 3D sequence. The "most linear" dUTE used the shortest possible first and second echoes. A longer second echo for the dUTE sequence would give steeper concentration sensitivity comparable to the VIBE sequence, but introduces the disadvantages of loss of contrast as the intensity of bone and muscle will also change between the two echoes and subtraction errors may occur from the increased susceptibility artifact at longer TE. The $T_{1}$ effect observed at low concentrations for the VIBE sequence will also start coming into play, destroying the monotonic increase leading to reduced linearity. A longer TE(1) (UTE) loses the advantages of this sequence in detecting the shortest possible $T_{2}$. The earliest possible 


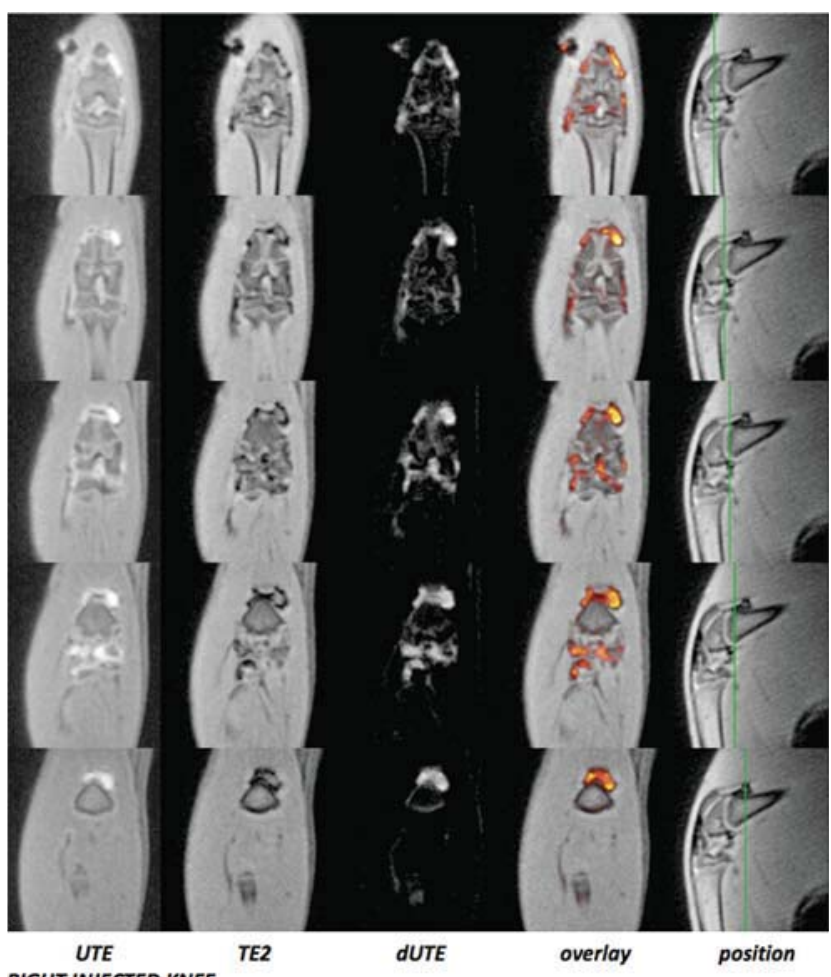

RIGHT INJECTED KNEE

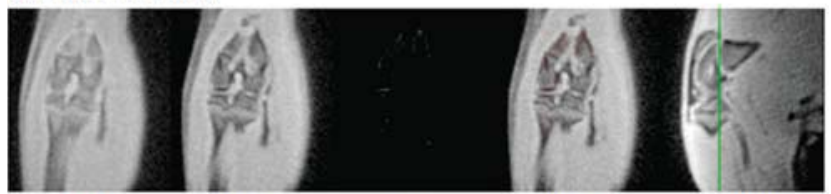

UTE

dUTE

overlay

position

LEFT CONTROL KNEE

FIG. 3. In vivo results at $50 \mu \mathrm{g} / \mathrm{mL}$ concentration. Iron oxide at 50 $\mu \mathrm{L}$ injected, UTE, TE(2), dUTE, dUTE fusion on anatomy and sagittal for localization. The region of synovial iron is clearly delineated. Several example slices are given throughout the knee. Last image row shows also the contralateral knee without injection. Red is low iron signal, through yellow to white for high iron concentration.

TE(2) was chosen, with "in-phase" fat/water to minimize artifacts and maximize the linear $T_{1} / T_{2}$ interplay of dUTE. Fat sat was applied to reduce any potential fat/ water artifacts.

The nonlinearity at high concentrations may be because, even at this short TE(1) we are not at "zero" TE and at high enough concentrations some signal loss starts to come into the effect and the first echo sequence. The linear signal is a balance in $T_{1}$ and $T_{2}$ effects between the two echoes in the difference image. This equilibrium starts to break down at very high concentrations, which are, fortunately, well above any uptake that would result from systemic injection in a biomarker study. The signal response is therefore still linear over the appropriate concentration range.

In addition to the concentration effects, an important consideration is contrast. Too much "background" tissue signal decay occurs with a longer TE(2) reducing contrast between iron regions. The use of the difference image, with low signal in all but the region of iron, makes use of SNR difficult as noise and background tissue are of the same order of magnitude. Subtraction also gives the consistent comparison between scans similar to taking an SNR ratio. Because of the sharp coil profile of the loop coil slight changes in position could cause possible difference in SNR in the exact region of iron uptake, although efforts were made with positioning to minimize this.

This important contrast factor gained by the background suppression in the dUTE sequence enables the distinction of synovial iron signal intensity from tissues (muscle and bone) and noise, unlike any of the classical sequences compared, or the simple single echo of the UTE acquisition. This is illustrated by the in vivo tests and results show separation of all tissues from regions of iron uptake. This guaranteed separation of tissues at all concentrations, however, must be taken in context with the monotonic dynamic of the concentration effect. In vivo distribution is not uniform, hence the spread of values of the three injected animals in each group. The distribution of actual concentration may vary in vivo due to diffusion effects as discussed below. The phantom studies, however, allow calibration of any concentration effects and show how even low concentrations can be detected. This opens the way for automatic quantification of signal purely from iron oxide. Contrast is higher between iron and other tissues for dUTE than on classical sequences with several hypointense tissues. The short echoes of the UTE and TE(2) images mean these give a truer representation of the size of the region of iron uptake as the sequence is less affected by susceptibility artifacts (30).

However, in vivo uptake is not uniform, hence the spread of values of the three injected animals in each group. Because of, for example, diffusion of the injected solution, the same injected concentration may result in differences in local concentration. Results from ANOVA confirm the advantages dUTE gives in terms of contrast and quantifiable iron oxide signal. Following on from this model, studies involving clinically relevant injection routes and timings can now be realized based on the results of this phantom study and the feasibility tests of known concentrations in vivo. The phantom studies, however, allow calibration of any concentration effects and show how even low concentrations can be detected.

One limitation of the study is that the actual quantification of iron is made before injection. Because of possible diffusion, the actual quantity in the image may be slightly lower than that injected. Interference from the skin and penetration depth did not allow optical calibration from the fluorophore (as well as the fact that free fluorophore in the agent may give an overestimated iron oxide concentration). Another option would have been to aspirate back the solution from the joint after imaging although we believe that losses here may be more than any small diffusive effect due to imaging immediately after injection. If the injection was not in the capsule, it has been observed that no signal is seen. We can therefore assume that the signal quantified results from complete successful injection of the contrast agent solution. 

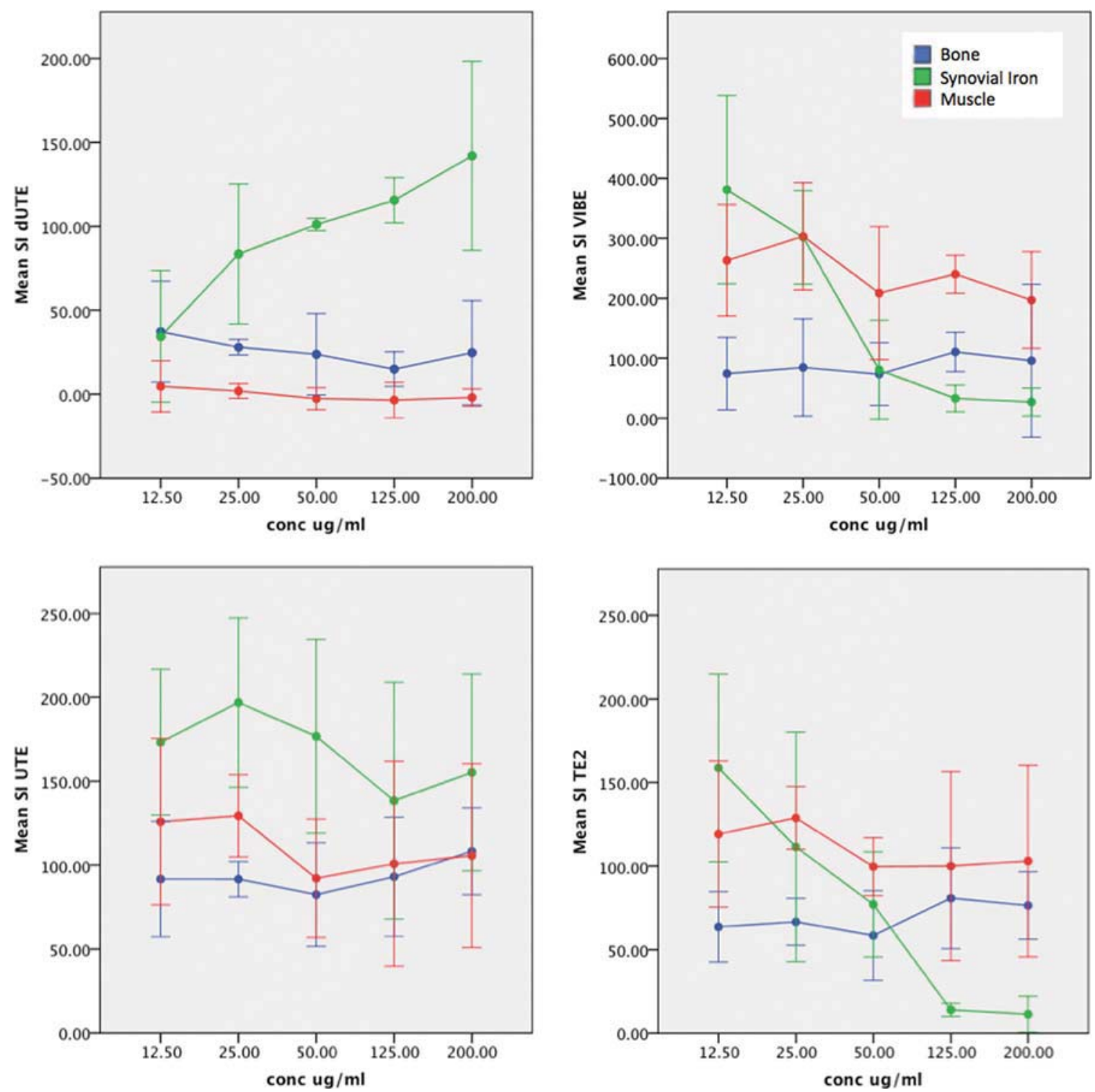

FIG. 4. In vivo results showing synovial iron signal emphasizing both the sensitivity to concentration from the slope and the contrast due to the separation of the iron data from the muscle/bone data. Non-normalized signal intensity of synovial iron region as a function of concentration $(\mu \mathrm{g} / \mathrm{mL})$ of solution injected intra-articular into the knee joint of healthy rats. Signal intensity on MRI is measured immediately after injection of $50 \mu \mathrm{L}$ of solution. Top row, dUTE and VIBE; bottom row, the individual echoes for creating dUTE (UTE and TE2). Each plot shows the effect of injected concentration on the signal intensity in the region of uptake as well as the signal for muscle and bone in the same animals. Error bars $95 \%$. [Color figure can be viewed in the online issue, which is available at wileyonlinelibrary.com.]

Three-dimensional isotropic resolution allows reconstruction of planes for easy registration of images with other sequences or for registration with, for example, histology slides. Further advantage over the highly iron-sensitive long TE sequences is that the acquisition time for 3D dUTE does not become prohibitively long. Also, in the eventual application of this method in arthritic joints, the competition between hyperintense edema and hypointense synovial iron in the $T_{2}$ STIR sequence in the same region of the diseased knee would make quantitative interpretation difficult. The $\mathrm{T}_{2}$ STIR sequences may be of interest for the very low dose of iron oxides particles, but it has the disadvantages of saturating to zero signal intensity at very low concentration of iron, and in a reasonable scan time it is only possible to acquire a $2 \mathrm{D}$ multislice, therefore not allowing isotropic high resolution. For very low dose of iron, the direct analysis of the UTE images may be of interest, and a comparison with STIR remains to be done. Its main clinical application relevant to future work in this project is detection of edema.

In conclusion, IA injections of IONs were compared with a concentration phantom using the dUTE sequence that gives a positive, unambiguous synovial iron signal and monotonic increasing concentration response over a wide concentration range with limited susceptibility artifacts and high contrast to all other tissues. 


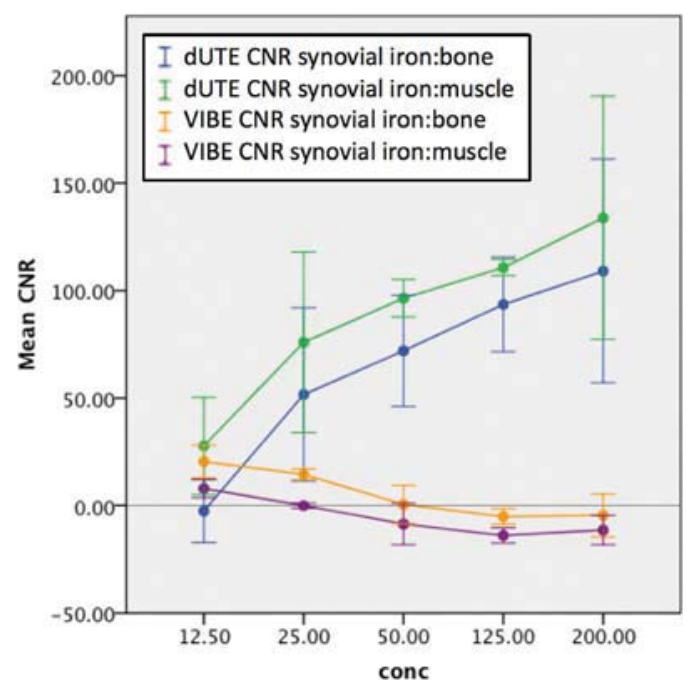

FIG. 5. Contrast-to-noise (difference between synovial iron and other tissue, normalized by noise background signal for the appropriate sequence) between synovial iron and other tissues is high and positive at all concentrations for the dUTE sequence compared with VIBE. Values are shown as mean of the three animals per group with $95 \%$ error bars. [Color figure can be viewed in the online issue, which is available at wileyonlinelibrary.com.]

\section{REFERENCES}

1. Butoescu N, Jordan O, Petri-Fink A, Hofmann H, Doelker E Co-encapsulation of dexamethasone 21-acetate and SPIONs into biodegradable polymeric microparticles designed for intra-articular delivery. J Microencapsul 2008;25:339-350.

2. Xie J, Lee S, Chen X. Nanoparticle-based theranostic agents. Adv Drug Delivery Rev 2010;62:1064-1079.

3. Beckmann N, Falk R, Zurbrugg S, Dawson J, Engelhardt P. Macrophage infiltration into the rat knee detected by MRI in a model of antigen-induced arthritis. Magn Reson Med 2003;49:1047-1055.

4. Evgenov NV, Medarova Z, Pratt J, Pantazopoulos P, Leyting S, Bonner-Weir S, Moore A. In vivo imaging of immune rejection in transplanted pancreatic islets. Diabetes 2006;55:2419-2428.

5. Medarova Z, Moore A. MRI as a tool to monitor islet transplantation. Nat Rev Endocrinol 2009;5:444-452.

6. Lee ES, Chan J, Shuter B, Tan LG, Chong MS, Ramachandra DL, Dawe GS, Ding J, Teoh SH, Beuf O, Briguet A, Tam KC, Choolani M, Wang SC. Microgel iron oxide nanoparticles for tracking human fetal mesenchymal stem cells through magnetic resonance imaging. Stem Cells 2009;27:1921-1931.

7. Ris F, Lepetit-Coiffe M, Meda P, Crowe LA, Toso C, Armanet M, Niclauss N, Parnaud G, Giovannoni L, Bosco D, Morel P, Vallee JP, Berney T. Assessment of human islet labeling with clinical grade iron nanoparticles prior to transplantation for graft monitoring by MRI. Cell Transplant 2010;19:1573-1585.

8. Montet-Abou K, Daire JL, Hyacinthe JN, Jorge-Costa M, Grosdemange K, Mach F, Petri-Fink A, Hofmann H, Morel DR, Vallee JP, Montet X. In vivo labelling of resting monocytes in the reticuloendothelial system with fluorescent iron oxide nanoparticles prior to injury reveals that they are mobilized to infarcted myocardium. Eur Heart J 2010; 31:1410-1420.

9. Marzola P, Longoni B, Szilagyi E, Merigo F, Nicolato E, Fiorini S, Paoli GT, Benati D, Mosca F, Sbarbati A. In vivo visualization of transplanted pancreatic islets by MRI: comparison between in vivo, histological and electron microscopy findings. Contrast Media Mol Imaging 2009;4:135-142.

10. Toso C, Vallee JP, Morel P, Ris F, Demuylder-Mischler S, LepetitCoiffe M, Marangon N, Saudek F, James Shapiro AM, Bosco D, Berney T. Clinical magnetic resonance imaging of pancreatic islet grafts after iron nanoparticle labeling. Am J Transplant 2008;8:701-706.

11. Stuber M, Gilson WD, Schar M, Kedziorek DA, Hofmann LV, Shah S Vonken EJ, Bulte JW, Kraitchman DL. Positive contrast visualization of iron oxide-labeled stem cells using inversion-recovery with ON- resonant water suppression (IRON). Magn Reson Med 2007;58: 1072-1077.

12. Dahnke H, Liu W, Herzka D, Frank JA, Schaeffter T. Susceptibility gradient mapping (SGM): a new postprocessing method for positive contrast generation applied to superparamagnetic iron oxide particle (SPIO)-labeled cells. Magn Reson Med 2008;60:595-603.

13. Liu W, Dahnke H, Jordan EK, Schaeffter T, Frank JA. In vivo MRI using positive-contrast techniques in detection of cells labeled with superparamagnetic iron oxide nanoparticles. NMR Biomed 2008;21: $242-250$.

14. Mills PH, Wu YJ, Ho C, Ahrens ET. Sensitive and automated detection of iron-oxide-labeled cells using phase image cross-correlation analysis. Magn Reson Imaging 2008;26:618-628.

15. Seevinck PR, de Leeuw H, Bos C, Bakker CJ. High positive contrast generation of a subvoxel susceptibility deviation in ultrashort TE (UTE) radial center-out imaging at 3T. Proc Int Soc Magn Reson Med 2009;868.

16. Diwoky C, Reinisch A, Gross D, Lehmann V, Strunk D, Stollberger R. UTE imaging for single cell detection with positive contrast. Proc Int Soc Magn Reson Med 2009;804.

17. Girard OM, Du J, Mattrey RF. Investigation of SPIO T1-signature: positive contrast using ultrashort TE imaging. Proc Int Soc Magn Reson Med 2009;2769.

18. Gatehouse PD, Bydder GM. Magnetic resonance imaging of short T2 components in tissue. Clin Radiol 2003;58:1-19.

19. Robson MD, Gatehouse PD, Bydder M, Bydder GM. Magnetic resonance: an introduction to ultrashort TE (UTE) imaging. J Comput Assist Tomogr 2003;27:825-846.

20. Crowe LA, Wang YX, Gatehouse PD, Tessier J, Waterton J, Robert P, Bydder G, Firmin DN. Ex vivo mr imaging of atherosclerotic rabbit aorta labelled with USPIO-enhancement of iron loaded regions in UTE imaging. Proc Int Soc Magn Reson Med 2005;115.

21. Crowe LA, Ris F, Nielles-Vallespin S, Speier P, Kocher M, Masson S, Toso C, Bosco D, Berney T, Vallee J-P. Comparison of rate of islet loss in syngeneic, allogeneic and xenogeneic grafts in rat using quantification of iron oxide labeled islet cells by 3D radial UTE MRI. Proc Int Soc Magn Reson Med 2010:209.

22. Crowe LA, Ris F, Nielles-Vallespin S, Speier P, Masson S, Armanet M, Morel P, Toso C, Bosco D, Berney T, Vallee JP. A novel method for quantitative monitoring of transplanted islets of langerhans by positive contrast magnetic resonance imaging. Am J Transplant 2011; 11:1158-1168.

23. Nielles-Vallespin S, Weber MA, Bock M, Bongers A, Speier P, Combs SE, Wohrle J, Lehmann-Horn F, Essig M, Schad LR. 3D radial projection technique with ultrashort echo times for sodium MRI: clinical applications in human brain and skeletal muscle. Magn Reson Med 2007;57:74-81.

24. Larson PZ, Gurney PT, Nishimura DG. Anisotropic field-of-views in radial imaging. IEEE Trans Med Imaging 2008;27:47-57.

25. Cunningham CH, Arai T, Yang PC, McConnell MV, Pauly JM, Conolly SM. Positive contrast magnetic resonance imaging of cells labeled with magnetic nanoparticles. Magn Reson Med 2005;53: 999-1005.

26. Thorek DL, Chen AK, Czupryna J, Tsourkas A. Superparamagnetic iron oxide nanoparticle probes for molecular imaging. Ann Biomed Eng 2006;34:23-38.

27. Le Renard PE, Jordan O, Faes A, Petri-Fink A, Hofmann H, Rufenacht D, Bosman F, Buchegger F, Doelker E. The in vivo performance of magnetic particle-loaded injectable, in situ gelling, carriers for the delivery of local hyperthermia. Biomaterials 2010;31:691-705.

28. Chastellain M, Petri A, Hofmann H. Particle size investigations of a multistep synthesis of PVA coated superparamagnetic nanoparticles. J Colloid Interface Sci 2004;278:353-360.

29. Steitz B, Salaklang J, Finka A, O’Neil C, Hofmann H, Petri-Fink A. Fixed bed reactor for solid-phase surface derivatization of superparamagnetic nanoparticles. Bioconjug Chem 2007;18:1684-1690.

30. Tyler DJ, Robson MD, Henkelman RM, Young IR, Bydder GM. Magnetic resonance imaging with ultrashort TE (UTE) PULSE sequences: technical considerations. J Magn Reson Imaging 2007;25:279-289.

31. Butoescu N, Jordan O, Burdet P, Stadelmann P, Petri-Fink A, Hofmann H, Doelker E. Dexamethasone-containing biodegradable superparamagnetic microparticles for intra-articular administration: physicochemical and magnetic properties, in vitro and in vivo drug release. Eur J Pharm Biopharm 2009;72:529-538. 